\title{
NUMERICAL RADIUS-ATTAINING OPERATORS ON $C(K)$
}

\author{
CARMEN SILVIA CARDASSI ${ }^{1}$
}

\begin{abstract}
Using a construction due to Johnson and Wolfe, we show that the numerical radius-attaining operators from $C(K)$ into itself are dense in the space of all operators, where $K$ is a compact Hausdorff space.
\end{abstract}

Let $X$ be a Banach space, $L(X)$ the Banach space of bounded linear operators from $X$ into $X$, and $\operatorname{NRA}(X)$ the subset of $L(X)$ consisting of the numerical radius-attaining operators.

Berg and Sims [1] have proved the "Bishop-Phelps type" result that $\operatorname{NRA}(X)$ is dense in $L(X)$ when $X$ is uniformly convex. Elsewhere we have shown the same to be so for $X$ being $c_{0}, l_{1}, L_{1}(\mu)$ or a uniformly smooth space.

In this note we consider the case of $X=C(K)$, the space of continuous real-valued functions on the compact Hausdorff space $K$. Following the lead of Johnson and Wolfe [3], we again show that $\operatorname{NRA}(C(K))$ is dense in $L(C(K))$.

We still do not know of any $X$ for which $\operatorname{NRA}(X)$ is not dense in $L(X)$. It may be that Lindenstrauss's example, using a renorming of $c_{0}$, for which the norm-attaining operators are not dense in $L(X)$ [4] also serves in the present setup, but we have not yet found that to be so.

We introduce initially some definitions and notations.

We define the numerical radius of a bounded linear operator $T: X \rightarrow X$, denoted by $v(T)$, by

$$
v(T)=\sup \left\{\left|x^{*}(T x)\right|:\left(x, x^{*}\right) \in \Pi(X)\right\},
$$

where $\Pi(X)=\left\{\left(x, x^{*}\right) \in X \times X^{*}:\left\|x^{*}\right\|=\|x\|=x^{*}(x)=1\right\}$.

We say that $T$ attains its numerical radius if there is $\left(x_{0}, x_{0}^{*}\right) \in \Pi(X)$ such that $v(T)=\left|x_{0}^{*}\left(T x_{0}\right)\right|$, and we denote the set of numerical radius-attaining operators by $\operatorname{NRA}(X)$.

If $K$ is a compact Hausdorff space and $X$ is a Banach space, we denote by $C_{\mathrm{w}}\left(K, X^{*}\right)$ the Banach space of continuous functions $F: K \rightarrow X^{*}$, where $X^{*}$ is equipped with its $\mathrm{w}^{*}$-topology, with the norm $\|F\|=\sup \{\|F(t)\|: t \in K\}$.

It is a well-known result that $C_{\mathrm{w}^{*}}\left(K, X^{*}\right)$ can be identified, isomorphically and isometrically, with the space $L(X, C(K))$ of all bounded linear operators from $X$ into $C(K)$, the identification being given by

$$
(T x)(t)=F(t)(x), \quad \forall t \in K, \forall x \in X,
$$

where $T \in L(X, C(K))[2$, p. 490].

Received by the editors August 16, 1984.

1980 Mathematics Subject Classification. Primary 47B99, 46E15.

${ }^{1}$ This work was partially supported by FAPESP (Săo Paulo, Brasil) and was done during a visit to Kent State University (Kent, Ohio). 
$M(K)$ denotes the space of regular Borel measures on $K$, with the norm of the variation, and is identified with $C(K)^{*}$.

In our case we will use the identification of $L(C(K))$ with $C_{\mathrm{w}^{*}}(K, M(K))$.

For the proof of the result announced in the abstract we need several lemmas.

Lemma 1. Given $F \in C_{\mathrm{w}^{*}}(K, M(K)), \varepsilon>0, f \in C(K), t_{0} \in K$ and an open set $V \subset K$, there is $U$, an open neighborhood of $t_{0}$, such that

(i) $|F(t)|(V) \geqslant\left|F\left(t_{0}\right)\right|(V)-\varepsilon, \forall t \in U$;

(ii) $F(t)(f) \geqslant F\left(t_{0}\right)(f)-\varepsilon, \forall t \in U$.

Proof. First we show that the function $\nu \in M(K) \mapsto|\nu|(V) \in \mathbf{R}$ is lower semicontinuous, where $M(K)$ has its w $^{*}$-topology.

In fact, if $\nu_{0} \in M(K)$, by Hahn decomposition and regularity of $\nu_{0}$ we can choose disjoint compact sets $K^{+}$and $K^{-}$, contained in $V$, such that $\left|\nu_{0}\right|\left(K^{+}\right)=\nu_{0}\left(K^{+}\right)$, $\left|\nu_{0}\right|\left(K^{-}\right)=-\nu_{0}\left(K^{-}\right)$and $\left|\nu_{0}\right|\left(V \backslash K^{+} \cup K^{-}\right)<\varepsilon / 3$.

Since $K$ is compact Hausdorff, we can choose $f_{0} \in C(K)$ with $\left|f_{0}(t)\right| \leqslant 1$, $\forall t \in K,\left.f_{0}\right|_{K^{+}}=1,\left.f_{0}\right|_{K^{-}}=-1$ and $\left.f_{0}\right|_{K \backslash V}=0$.

Let $A=\left\{\nu \in M(K):\left|\nu\left(f_{0}\right)-\nu_{0}\left(f_{0}\right)\right|<\varepsilon / 3\right\}$. Then $A$ is a $w^{*}$-neighborhood of $\nu_{0}$, and if $\nu \in A$ we have

$$
\begin{aligned}
|\nu|(V) & \geqslant \int_{V} f_{0} d|\nu| \geqslant\left|\int_{V} f_{0} d \nu\right|=\left|\nu\left(f_{0}\right)\right|>\left|\nu_{0}\left(f_{0}\right)\right|-\frac{\varepsilon}{3} \\
& =\left|\int_{V} f_{0} d \nu_{0}\right|-\frac{\varepsilon}{3} \geqslant \int_{V} f_{0} d \nu_{0}-\frac{\varepsilon}{3} \\
& =\int_{K^{+}} d \nu_{0}-\int_{K^{-}} d \nu_{0}+\int_{V \backslash K^{+} \cup K^{-}} f_{0} d \nu_{0}-\frac{\varepsilon}{3} \\
& >\nu_{0}\left(K^{+}\right)-\nu_{0}\left(K^{-}\right)-\frac{\varepsilon}{3}-\frac{\varepsilon}{3}>\left|\nu_{0}\right|(V)-\frac{\varepsilon}{3}-\frac{\varepsilon}{3}-\frac{\varepsilon}{3}=\left|\nu_{0}\right|(V)-\varepsilon .
\end{aligned}
$$

Since $F \in C_{\mathrm{w}^{*}}(K, M(K))$, the composite function $t \in K \mapsto|F(t)|(V)$ is also lower semicontinuous. Thus there is an open neighborhood $U_{1}$ of $t_{0}$ such that for $t \in U_{1}$ we have $|F(t)|(V)>\left|F\left(t_{0}\right)\right|(V)-\varepsilon$.

Also, given $B=\left\{\nu \in M(K):\left|\nu(f)-F\left(t_{0}\right)(f)\right|<\varepsilon\right\}$, which is a w*-neighborhood of $F\left(t_{0}\right) \in M(K)$, there is $U_{2}$, an open neighborhood of $t_{0}$ such that for $t \in U_{2}$ we have $F(t) \in B$, since $F \in C_{\mathrm{w}^{*}}(K, M(K))$. Then if $t \in U_{2}$ we have $F(t)(f) \geqslant$ $F\left(t_{0}\right)(f)-\varepsilon$.

Letting $U=U_{1} \cap U_{2}$ we have that $U$ is an open neighborhood of $t_{0}$ and, for $t \in U$, (i) and (ii) hold.

Lemma 2. Given $F \in C_{\mathbf{w}^{*}}(K, M(K))$ and $\varepsilon>0$, there are $f_{0} \in C(K),\left\|f_{0}\right\|_{\infty}=1$ and $t_{0} \in K$ such that $F\left(t_{0}\right)\left(f_{0}\right)>\|F\|-\varepsilon$ and $\left|f_{0}\left(t_{0}\right)\right|=1$.

Proof. Let $t_{0} \in K$ be such that $\left|F\left(t_{0}\right)\right|(K)>\|F\|-\varepsilon / 3$.

For simplicity let us set $\mu_{0}=F\left(t_{0}\right)$. Then $\left|\mu_{0}\right|(K)>\|F\|-\varepsilon / 3$.

Using Hahn decomposition and regularity of $\mu_{0}$, we can choose disjoint compact sets $K^{+}$and $K^{-}$such that $\left|\mu_{0}\right|\left(K^{+}\right)=\mu_{0}\left(K^{+}\right),\left|\mu_{0}\right|\left(K^{-}\right)=-\mu\left(K^{-}\right)$and

$$
\left|\mu_{0}\right|\left(K \backslash K^{+} \cup K^{-}\right)<\varepsilon / 3 \text {. }
$$

Then $\mu_{0}\left(K^{+}\right)-\mu_{0}\left(K^{-}\right)>\left|\mu_{0}\right|(K)-\varepsilon / 3$. 
Case I. $t_{0} \in K^{+} \cup K^{-}$.

Since $K$ is compact Hausdorff, we can choose $f_{0} \in C(K),\left|f_{0}(t)\right| \leqslant 1, \forall t \in K$, $\left.f_{0}\right|_{K^{+}}=1$, and $\left.f_{0}\right|_{K^{-}}=-1$.

Then

$$
\begin{aligned}
F\left(t_{0}\right)\left(f_{0}\right) & =\int_{K} f_{0} d \mu_{0}=\int_{K^{+}} d \mu_{0}-\int_{K^{-}} d \mu_{0}+\int_{K \backslash K^{+} \cup K^{-}} f_{0} d \mu_{0} \\
& =\mu_{0}\left(K^{+}\right)-\mu_{0}\left(K^{-}\right)+\int_{K \backslash K^{+} \cup K^{-}} f_{0} d \mu_{0} .
\end{aligned}
$$

Since

$$
\left|\int_{K \backslash K^{+} \cup K^{-}} f_{0} d \mu_{0}\right| \leqslant\left|\mu_{0}\right|\left(K \backslash K^{+} \cup K^{-}\right)<\varepsilon / 3
$$

we get

$$
\begin{aligned}
F\left(t_{0}\right)\left(f_{0}\right) & >\mu_{0}\left(K^{+}\right)-\mu_{0}\left(K^{-}\right)-\varepsilon / 3>\left|\mu_{0}\right|(K)-\varepsilon / 3-\varepsilon / 3 \\
& >\|F\|-\varepsilon / 3-\varepsilon / 3-\varepsilon / 3=\|F\|-\varepsilon .
\end{aligned}
$$

Obviously in this case we have $\left|f_{0}\left(t_{0}\right)\right|=1$, since $t_{0} \in K^{+} \cup K^{-}$and $\left.\left|f_{0}\right|\right|_{K^{+} \cup K^{-}}=1$. Case II. $t_{0} \notin K^{+} \cup K^{-}$.

Since $K^{+} \cup\left\{t_{0}\right\}$ and $K^{-}$are again disjoint compact sets, let $f_{0} \in C(K)$ be such that $\left|f_{0}(t)\right| \leqslant 1, \forall t \in K,\left.f_{0}\right|_{K^{+} \cup\left\{t_{0}\right\}}=1$ and $\left.f_{0}\right|_{K^{-}}=-1$.

As in Case I we have $F\left(t_{0}\right)\left(f_{0}\right)>\|F\|-\varepsilon$ and $f_{0}\left(t_{0}\right)=1$, by definition of $f_{0}$.

As an easy consequence we have

Corollary 3. $v(T)=\|T\|, \forall T \in C(K)$.

The next lemma is a modification of a result of Johnson and Wolfe [3].

LEMMA 4. Given $F \in C_{\mathrm{w}^{*}}(K, M(K))$ and $\varepsilon>0$, there are open subsets $V_{1}$ and $V_{2}$ of $K$, with $\bar{V}_{1} \cap \bar{V}_{2}=\varnothing, V_{2} \neq \varnothing$, and there are $f_{1} \in C(K),\left\|f_{1}\right\|_{\infty}=1$, and $F_{1} \in$ $C_{\mathrm{w}^{*}}(K, M(K))$ such that

(i) $\left|f_{1}(t)\right|=1, \forall t \in K \backslash V_{1}$;

(ii) $\left|F_{1}(t)\right|\left(V_{1}\right)=0, \forall t \in V_{2}$;

(iii) $F_{1}(t)\left(f_{1}\right)>\left\|F_{1}\right\|-\varepsilon, \forall t \in V_{2}$;

(iv) $\left\|F-F_{1}\right\|<\varepsilon$.

Proof. Let $t_{0} \in K$ be such that $\left|F\left(t_{0}\right)\right|(K)>\|F\|-\varepsilon / 4$.

Using $\left(B^{+}, B^{-}\right)$a Hahn decomposition of $K$ for $\mu_{0}=F\left(t_{0}\right)$ and the regularity of $\mu_{0}$, choose $K^{+} \subset B^{+}$and $K^{-} \subset B^{-}$compact sets such that

$$
\mu_{0}\left(K^{+}\right)-\mu_{0}\left(K^{-}\right)>\left|\mu_{0}\right|(K)-\varepsilon / 4>\|F\|-\varepsilon / 2 .
$$

As in the proof of Lemma 2, let $f_{0} \in C(K),\left\|f_{0}\right\|_{\infty}=1$, be such that $\left.f_{0}\right|_{K^{+}}=1$, $\left.f_{0}\right|_{K^{-}}=-1$ and $\left.\left|f_{0}\right|\right|_{K^{+} \cup K^{-} \cup\left\{t_{0}\right\}}=1$.

For each $\alpha \in] 0,1\left[\right.$, let $A_{\alpha}=\left\{t \in K:\left|f_{0}(t)\right|<\alpha\right\}$.

Case I. $\left.A_{\alpha}=\varnothing, \forall \alpha \in\right] 0,1[$.

In this case, $\left|f_{0}(t)\right|=1, \forall t \in K$. Define $f_{1}=f_{0}, V_{1}=\varnothing$ and $F_{1}=F$. Then (i) and (ii) hold for $t \in K$ and (iv) also is satisfied. 
Moreover,

$$
F_{1}\left(t_{0}\right)\left(f_{1}\right)=F\left(t_{0}\right)\left(f_{0}\right) \geqslant \mu_{0}\left(K^{+}\right)-\mu_{0}\left(K^{-}\right)-\varepsilon / 4>\|F\|-3 \varepsilon / 4 .
$$

By Lemma 1 , using $\varepsilon / 4$, there is $V_{2} \subset K$, an open neighborhood of $t_{0}$, such that $F_{1}(t)\left(f_{1}\right) \geqslant F_{1}\left(t_{0}\right)\left(f_{1}\right)-\varepsilon / 4, \forall t \in V_{2}$.

Then $F_{1}(t)\left(f_{1}\right) \geqslant\|F\|-\varepsilon=\left\|F_{1}\right\|-\varepsilon, \forall t \in V_{2}$ and (iii) holds.

Obviously, $V_{2} \neq \varnothing$ and $\bar{V}_{1} \cap \bar{V}_{2}=\varnothing$, and we are done.

Case II. There is $\left.\alpha_{0} \in\right] 0,1\left[\right.$ with $A_{\alpha_{0}} \neq \varnothing$.

In this case let $\beta_{0}$ be such that $\alpha_{0}<\beta_{0}<1$.

Define $V_{1}=\left\{t \in V:\left|f_{0}(t)\right|<\alpha_{0}\right\}=A_{\alpha_{0}}$ and $W=\left\{t \in K:\left|f_{0}(t)\right|>\beta_{0}\right\}$. Then $V_{1}$ and $W$ are open sets, $\bar{V}_{1} \cap W=\varnothing$ and $\left\{t_{0}\right\} \cup K^{+} \cup K^{-} \subset W$.

Since $A_{\alpha_{0}} \neq \varnothing$, fix $t_{1} \in V_{1}$ and choose $f_{1}, g \in C(K),\left|f_{1}(t)\right| \leqslant 1,0 \leqslant g(t) \leqslant 1$, $\forall t \in K$, such that

$$
f_{1}(t)=\left\{\begin{array}{ll}
1 & \text { if } t \in \overline{\left(K \backslash V_{1}\right) \cap B^{+}}, \\
-1 & \text { if } t \in \overline{\left(K \backslash V_{1}\right) \cap B^{-}}, \\
0 & \text { if } t=t_{1}
\end{array} \quad \text { and } \quad g(t)= \begin{cases}0 & \text { if } t \in \bar{W} \\
1 & \text { if } t \in \bar{V}_{1}\end{cases}\right.
$$

Then (i) holds and since

$$
\left[(1-g) f_{1}\right](t)=1 \quad \text { if } t \in K^{+}, \quad\left[(1-g) f_{1}\right](t)=-1 \quad \text { if } t \in K^{-}
$$

and

$$
\left|\left[(1-g) f_{1}\right](t)\right| \leqslant 1 \text { if } t \in\left(B^{+} \backslash K^{+}\right) \cup\left(B^{-} \backslash K^{-}\right),
$$

we have

$$
\begin{aligned}
F\left(t_{0}\right)\left((1-g) f_{1}\right)= & \int_{K}(1-g) f_{1} d \mu_{0} \\
= & \int_{K^{+}}(1-g) f_{1} d \mu_{0}+\int_{K^{-}}(1-g) f_{1} d \mu_{0} \\
& +\int_{\left(B^{+} \backslash K^{+}\right) \cup\left(B^{-} \backslash K^{-}\right)}(1-g) f_{1} d \mu_{0} \\
\geqslant & \mu_{0}\left(K^{+}\right)-\mu_{0}\left(K^{-}\right)-\left|\mu_{0}\right|\left(\left(B^{+} \backslash K^{+}\right) \cup\left(B^{-} \backslash K^{-}\right)\right) \\
\geqslant & \left|\mu_{0}\right|(K)-\varepsilon / 4-\varepsilon / 4>\|F\|-3 \varepsilon / 4 .
\end{aligned}
$$

By Lemma 1 , using $\varepsilon / 4$, there is $U \subset K$ an open neighborhood of $t_{0}$ such that for each $t \in U$,

$$
F(t)\left((1-g) f_{1}\right) \geqslant F\left(t_{0}\right)\left((1-g) f_{1}\right)-\varepsilon / 4>\|F\|-\varepsilon
$$

and

$$
|F(t)|(W) \geqslant\left|F\left(t_{0}\right)\right|(W)-\varepsilon / 4>\|F\|-\varepsilon .
$$

We can take $U \cap \bar{V}_{1}=\varnothing$, since $t_{0} \notin \bar{V}_{1}$. Let $V_{2} \subset U$ be an open set such that $t_{0} \in V_{2}$ and $\bar{V}_{2} \subset U$. In particular, $V_{2} \neq \varnothing$ and $\bar{V}_{1} \cap \bar{V}_{2}=\varnothing$.

Choose $h \in C(K),\|h\|_{\infty}=1, h(t)=1$ if $t \in \bar{V}_{2}$ and $h(t)=0$ if $t \in K \backslash U$ and define $F_{1}: K \rightarrow M(K)$ by $F_{1}(t)=[1-h(t) g] F(t), \forall t \in K$, which means

$$
F_{1}(t)(p)=F(t)([1-h(t) g] p), \quad \forall p \in C(K) \text {. }
$$


Since $g \in C(K), \quad F_{1}(t) \in M(K), \quad \forall t \in K$ and since $h \in C(K)$ and $F \in C_{\mathrm{w}^{*}}(K, M(K)), \quad F_{1} \in C_{\mathrm{w}^{*}}(K, M(K))$. Also $\left|F_{1}(t)\right|(K) \leqslant|F(t)|(K)$, since $\|1-h(t) g\|_{\infty} \leqslant 1, \forall t \in K$, and then $\left\|F_{1}\right\| \leqslant\|F\|$.

If $t \in V_{2}, h(t)=1$ and $F_{1}(t)=(1-g) F(t)$. Since $\left.g\right|_{\bar{V}_{1}}=1,\left|F_{1}(t)\right|\left(V_{1}\right)=0$ and (ii) holds. Also

$$
F_{1}(t)\left(f_{1}\right)=F(t)\left((1-g) f_{1}\right)>\|F\|-\varepsilon \geqslant\left\|F_{1}\right\|-\varepsilon
$$

and (iii) holds. For (iv), note that

$$
\left|F(t)-F_{1}(t)\right|(K)=|h(t) g F(t)|(K)=0 \quad \text { if } t \in K \backslash U,
$$

since $\left.h\right|_{K \backslash U}=0$ and

$$
\left|F(t)-F_{1}(t)\right|(K) \leqslant|g F(t)|(K) \quad \text { if } t \in U .
$$

But $\left.g\right|_{\bar{W}}=0$ and then

$$
\begin{aligned}
|g F(t)|(K) & =|g F(t)|(K \backslash \bar{W}) \leqslant|F(t)|(K \backslash \bar{W}) \\
& =|F(t)|(K)-|F(t)|(\bar{W}) \leqslant\|F\|-|F(t)|(W) \\
& <\|F\|-(\|F\|-\varepsilon)=\varepsilon \quad \text { if } t \in U .
\end{aligned}
$$

Then

$$
\left\|F-F_{1}\right\|=\sup \left\{\left|F(t)-F_{1}(t)\right|(K): t \in K\right\} \leqslant \varepsilon .
$$

The proof of the next lemma can be found in [3, Lemma 2.4].

Lemma 5. Let $F \in C_{\mathrm{w}^{*}}(K, M(K)), V_{1}, V_{2} \subset K$ open sets, $t_{0} \in V_{2}, f_{0} \in C(K)$, $\left\|f_{0}\right\|_{\infty}=1$, be such that
(a) $|F(t)|\left(V_{1}\right)=0, \forall t \in V_{2}$;
(b) $F\left(t_{0}\right)\left(f_{0}\right) \geqslant\|F\|-\varepsilon$;
(c) $\left|f_{0}(t)\right|=1, \forall t \in K \backslash V_{1}$.

Then for every $r>2 / 3$, there is $F_{1} \in C_{\mathrm{w}^{*}}(K, M(K))$, and there is $t_{1} \in V_{2}$ such that

(i) $\left|F_{1}(t)\right|\left(V_{1}\right)=0, \forall t \in V_{2}$;

(ii) $F_{1}\left(t_{1}\right)\left(f_{0}\right) \geqslant\left\|F_{1}\right\|-r \varepsilon$;

(iii) $\left\|F-F_{1}\right\|<r \varepsilon$.

THEOREM 6. $\overline{\operatorname{NRA}(C(K))}=L(C(K))$.

Proof. Let $T \in L(C(K))$ and $\varepsilon>0$ be given, and let $F \in C_{\mathrm{w}^{*}}(K, M(K))$ be the representative of $T$.

Take $2 / 3<r<1$ and apply Lemma 4 to get $F_{0} \in C_{\mathrm{w}^{*}}(K, M(K)), V_{1}, V_{2} \subset K$ open sets, $\bar{V}_{1} \cap \bar{V}_{2}=\varnothing, V_{2} \neq \varnothing, f_{0} \in C(K),\left\|f_{0}\right\|_{\infty}=1$, such that

(a) $\left|F_{0}(t)\right|\left(V_{1}\right)=0, \forall t \in V_{2}$;

(b) $F_{0}(t)\left(f_{0}\right)>\left\|F_{0}\right\|-\varepsilon(1-r), \forall t \in V_{2}$;

(c) $\left|f_{0}(t)\right|=1, \forall t \in K \backslash V_{1}$;

(d) $\left\|F-F_{0}\right\|<\varepsilon(1-r)$.

Choose $t_{0} \in V_{2}$ such that

(b') $F_{0}\left(t_{0}\right)\left(f_{0}\right)>\left\|F_{0}\right\|-\varepsilon(1-r)$, and let $\lambda=\left\|F_{0}\right\|-F_{0}\left(t_{0}\right)\left(f_{0}\right)$. Then $0 \leqslant \lambda<$ $\varepsilon(1-r)$. 
Case I. $\lambda=0$.

In this case, $\left\|F_{0}\right\|=F_{0}\left(t_{0}\right)\left(f_{0}\right)=\delta_{t_{0}}\left(T_{0} f_{0}\right)$, where $T_{0} \in L(C(K))$ corresponds to $F_{0}$.

Defining $\mu_{0}=\left(\operatorname{sgn} f_{0}\left(t_{0}\right)\right) \delta_{t_{0}}$, we have $\mu_{0}\left(f_{0}\right)=\left|f_{0}\left(t_{0}\right)\right|=1$, since $t_{0} \in V_{2}$ and $V_{1} \cap V_{2}=\varnothing$, and $\left|\mu_{0}\right|(K)=1$. Then $\left(f_{0}, \mu_{0}\right) \in \Pi(C(K))$.

Also we have $T_{0} \in \operatorname{NRA}(C(K))$, for

$$
\left|\mu_{0}\left(T_{0} f_{0}\right)\right|=\left|\delta_{t_{0}}\left(T_{0} f_{0}\right)\right|=\left\|F_{0}\right\|=\left\|T_{0}\right\| .
$$

From (d), $\left\|T-T_{0}\right\|=\left\|F-F_{0}\right\|<\varepsilon(1-r)<\varepsilon$, and we are done.

Case II. $\lambda>0$.

By definition of $\lambda$,

(b') $F_{0}\left(t_{0}\right)\left(f_{0}\right)=\left\|F_{0}\right\|-\lambda$.

Now (a), ( $\left.\mathrm{b}^{\prime \prime}\right)$ and (c) allow us to apply Lemma 5 and get $F_{1} \in C_{\mathrm{w}^{*}}(K, M(K))$ and $t_{1} \in V_{2}$ such that

$\left(\mathrm{a}_{1}\right)\left|F_{1}(t)\right|\left(V_{1}\right)=0, \forall t \in V_{2}$;

$\left(\mathrm{b}_{1}\right) F_{1}\left(t_{1}\right)\left(f_{0}\right) \geqslant\left\|F_{1}\right\|-r \lambda$;

$\left(\mathrm{d}_{1}\right)\left\|F_{0}-F_{1}\right\|<r \lambda$.

Again $\left(\mathrm{a}_{1}\right),\left(\mathrm{b}_{1}\right)$ and (c) allow us to apply Lemma 5 and get $F_{2} \in C_{\mathrm{w}^{*}}(K, M(K))$ and $t_{2} \in V_{2}$ such that

$\left(\mathrm{a}_{2}\right)\left|F_{2}(t)\right|\left(V_{1}\right)=0, \forall t \in V_{2}$;

$\left(\mathrm{b}_{2}\right) F_{2}\left(t_{2}\right)\left(f_{0}\right) \geqslant\left\|F_{2}\right\|-r^{2} \lambda$;

$\left(\mathrm{d}_{2}\right)\left\|F_{1}-F_{2}\right\|<r^{2} \lambda$.

Following in this way we get sequences $\left\{F_{n}\right\}$ in $C_{\mathrm{w}^{*}}(K, M(K))$ and $\left\{t_{n}\right\}$ in $V_{2}$ such that for each $n \in \mathbf{N}$,

$\left(\mathrm{b}_{n}\right) F_{n}\left(t_{n}\right)\left(f_{0}\right) \geqslant\left\|F_{n}\right\|-r^{n} \lambda$;

$\left(\mathrm{d}_{n}\right)\left\|F_{n-1}-F_{n}\right\| \leqslant r^{n} \lambda$.

Since $K$ is compact, $\left\{t_{n}\right\}$ has a subsequence convergent to some $\tilde{t} \in K$. But $t_{n} \in V_{2}, \forall n \in \mathbf{N}$ and then $\tilde{t} \in \bar{V}_{2}$. We still denote this subsequence by $\left\{t_{n}\right\}$.

On the other hand, if $m>n \geqslant 1$, by $\left(\mathrm{d}_{n}\right)$ it follows that

$$
\left\|F_{n}-F_{m}\right\| \leqslant \sum_{k=n+1}^{m}\left\|F_{k}-F_{k-1}\right\| \leqslant\left(\sum_{k=n+1}^{m} r^{k}\right) \lambda .
$$

Since $r<1$, this shows that $\left\{F_{n}\right\}$ is Cauchy in $C_{\mathrm{w}^{*}}(K, M(K))$ and so is $\left\{T_{n}\right\}$ in $L(C(K))$, where $T_{n}$ corresponds to $F_{n}, \forall n \in \mathbf{N}$.

Let $\tilde{T} \in L(C(K))$ be the limit of $\left\{T_{n}\right\}$ and $\tilde{F}$ its correspondent in $C_{\mathrm{w}^{*}}(K, M(K))$.

We have

$$
\begin{aligned}
\left\|T-T_{n}\right\| & \leqslant\left\|T-T_{0}\right\|+\left\|T_{0}-T_{n}\right\| \leqslant \varepsilon(1-r)+\left(\sum_{k=1}^{n} r^{k}\right) \lambda \\
& \leqslant \varepsilon(1-r)+\frac{r}{1-r} \varepsilon(1-r)=\varepsilon, \quad \forall n \in \mathbf{N} .
\end{aligned}
$$

From this it follows that $\|T-\tilde{T}\| \leqslant \varepsilon$.

It remains to show that $\tilde{T} \in \operatorname{NRA}(C(K))$. 
From $\left|\tilde{F}\left(t_{n}\right)\left(f_{0}\right)-F_{n}\left(t_{n}\right)\left(f_{0}\right)\right| \leqslant\left\|\tilde{F}-F_{n}\right\|$ and $\left(\mathrm{b}_{n}\right)$ we get

$$
\begin{aligned}
\tilde{F}\left(t_{n}\right)\left(f_{0}\right) & \geqslant F_{n}\left(t_{n}\right)\left(f_{0}\right)-\left\|\tilde{F}-F_{n}\right\| \geqslant\left\|F_{n}\right\|-r^{n} \lambda-\left\|\tilde{F}-F_{n}\right\| \\
& \geqslant\|\tilde{F}\|-r^{n} \lambda-2\left\|\tilde{F}-F_{n}\right\|, \quad \forall n \in \mathbf{N},
\end{aligned}
$$

and since

$$
\left\|\tilde{F}-F_{n}\right\| \leqslant\left(\sum_{k=n+1}^{\infty} r^{k}\right) \lambda=\frac{r^{n+1}}{1-r} \lambda<r^{n+1}, \quad \forall n \in \mathbf{N}
$$

we have

$$
\tilde{F}\left(t_{n}\right)\left(f_{0}\right) \geqslant\|\tilde{F}\|-r^{n} \varepsilon(1-r)-2 r^{n+1} \varepsilon=\|\tilde{F}\|-r^{n} \varepsilon(3-r) .
$$

Now, since $\tilde{F}$ is $\mathrm{w}^{*}$-continuous and $t_{n} \rightarrow \tilde{t}$ and $r^{n} \rightarrow 0$, we have

$$
\tilde{F}(\tilde{t})\left(f_{0}\right) \geqslant\|\tilde{F}\| \quad \text { or } \quad \delta_{\tilde{t}}\left(\tilde{T} f_{0}\right) \geqslant\|\tilde{T}\| \text {. }
$$

Also $\left|f_{0}(\tilde{t})\right|=\lim _{n \rightarrow \infty}\left|f_{0}\left(t_{n}\right)\right|=1$, since $f_{0}$ is continuous, $t_{n} \rightarrow \tilde{t}$ and $\left|f_{0}\left(t_{n}\right)\right|=1$, because $t_{n} \in V_{2}$ and $\bar{V}_{2} \cap \bar{V}_{1}=\varnothing$.

Defining $\tilde{\mu}=\left(\operatorname{sgn} f_{0}(\tilde{t})\right) \delta_{i}$, we have $\tilde{\mu} \in M(K),|\tilde{\mu}|(K)=1$, and $\tilde{\mu}\left(f_{0}\right)=\left|f_{0}(\tilde{t})\right|=$ 1. Then $\left(f_{0}, \tilde{\mu}\right) \in \Pi(C(K))$.

Since $\left|\tilde{\mu}\left(\tilde{T} f_{0}\right)\right|=\left|\delta_{i}\left(\tilde{T} f_{0}\right)\right| \geqslant\|\tilde{T}\|$ and $\|\tilde{T}\|=v(\tilde{T})$, by Corollary 3 , we get $\left|\tilde{\mu}\left(\tilde{T} f_{0}\right)\right|$ $\geqslant v(\tilde{T})$. But $v(\tilde{T}) \geqslant\left|\tilde{\mu}\left(\tilde{T} f_{0}\right)\right|$, and then $v(\tilde{T})=\left|\tilde{\mu}\left(\tilde{T} f_{0}\right)\right|$ and $\tilde{T} \in \operatorname{NRA}(C(K))$.

Acknowledgement. We are grateful to Professor Joseph Diestel for introducing us to the subject of this work and for his help during the preparation of this paper.

\section{REFERENCES}

1. I. D. Berg and B. Sims, Denseness of numerical radius attaining operators, J. Austral. Math. Soc. Ser. A 36 (1984), 130-133.

2. N. Dunford and J. T. Schwartz, Linear operators. I, Interscience, New York, 1958.

3. J. Johnson and J. Wolfe, Norm attaining operators, Studia Math. 65 (1979), 7-19.

4. J. Lindenstrauss, On operators which attain their norm, Israel J. Math. 1 (1963), 139-148.

Instituto de Matemática e Estatística, Universidade de São Paulo, C. P. 20.570 (Ag. Iguatemi), 01498-Săo PaUlo, SP-Brasil 